\title{
MÉTODO Y CONOCIMIENTO EN HISTORIA. INCIDENCIA EN LA CONSIDERACIÓN DE LA IDEA DE NACIÓN ${ }^{1}$
}

\author{
$\mathrm{M}^{\mathrm{a}}$ Isabel LAFUENTE GUANTES \\ Universidad de León
}

\begin{abstract}
RESUMEN: Se consideran dos orientaciones fundamentales del trabajo histórico: empírica y positiva, y sus metodologías: método de recuperación/método constructivo. Se analizan los problemas que están produciendo en el conocimiento histórico la introducción de los imaginarios y las TICs en relación con las nociones de estructura, orden y conexión. Se examinan dos formas metodológicas (tipos y modelos) y su capacidad para poder trabajar con los problemas de continuidad/discontinuidad en tanto afectan a la realidad histórica. Se aplican los análisis a la idea de nación.
\end{abstract}

PALABRAS CLAVE: Historia Empírica, Historia Total, Imaginario, Métodos, Conexión, Orden, Continuidad/Discontinuidad.

ABSTRACT: This paper examines two fundamental directions of the historical work: empirical and positivist, and their methodologies: Method of Recovery/Constructive Method. The problems produced in the historical knowledge by the introduction of the imaginary ones and the TICs in relation with the notions of structure, order, and connection are analyzed. Two methodological forms (types and models) and their ability to work with the problems of continuity / discontinuity are also considered, as far as they concern historical reality. These analyses are applied to the idea of nation.

KEYWORDS: Empirical History, Total History, Imaginary, Methods, Connection, Order, Continuity / Discontinuity.

\section{INTRODUCCIÓN}

La historia es una disciplina controvertida, lo que seguramente se debe a su tema propio: saber sobre el pasado. El principio de que el hombre necesita saber la verdad, pues de ello depende saber qué hacer, que justifica la necesidad del conocimiento, es también el que sirve de guía al trabajo histórico; en él se apoya la concepción de Tucídides de que la Historia reconstruye el pasado, cuya virtud se muestra en la afirmación de Vico de que la verdad está en lo hecho, pero también

\footnotetext{
${ }^{1}$ Recibido el 9 de septiembre y aceptado para su publicación el 25 de octubre de 2010.
} 
la afirmación de Cicerón de que la Historia enseña a los hombres cómo vivir. Considero que es en la convergencia de estas dos dimensiones: conocimiento de lo hecho y utilidad de ese conocimiento (enseñar a vivir), en la que radica una de las principales dificultades que afecta a la Historia, y al hacer del historiador, y del que necesariamente tiene que ocuparse la Filosofía y la Teoría y Metodología de la Historia.

La dificultad se centra en que la reconstrucción histórica implica la temporalidad de forma muy especial, lo que puede percibirse con claridad en la distinción aristotélica entre poesía e historia. Para Aristóteles la poesía y la historia coinciden en ser narraciones, pero lo propio de la primera es contar lo general, mientras que la segunda cuenta lo particular, y esto supone para el estagirita que la necesidad de lo narrado en poesía tiene que ver con lo posible (lo que podría haber sucedido o puede suceder), por lo que puede permitirse la licencia de narrar también según verosimilitud, mientras que la necesidad de lo narrado en historia tiene que ver con su realidad, por tanto, tiene que explicitarse la razón de la relación entre la narración y los datos. Aristóteles consideraba la poesía más científica que la historia, es decir, consideraba más filosófico lo necesarioverdadero-formal, que en relación con los hechos puede ser sólo verosímil, que lo necesario-verdadero-material; consideraba más filosófica una estructura formal, tan solo dotada de coherencia y lógica interna, que una estructura que, por afectar a los hechos, es una estructura lógico-material. Por ello, poesía e historia, según él, no se distinguen porque una escriba en verso y la otra en prosa, sino porque una atiende a lo general, y la otra a lo particular. ${ }^{2}$

A partir de la concepción aristotélica, vamos a considerar que atender a lo particular (individuo histórico), a lo que tiene que ver con unos hechos dados en espacios-tiempos reales, tiene una función inmediata: la de permitir comparaciones pertinentes que posibiliten saber sobre la situación de lo particular en diversas etapas o momentos históricos, y dar cuenta de cómo el discurrir temporal adquiere la forma de progreso. En este sentido vamos a distinguir las principales formas en que hoy ${ }^{3}$ se aborda el hacer histórico.

\section{LA HISTORIA EMPÍRICA: EL MÉTODO DE RECUPERACIÓN}

La primera forma, que suele denominarse Historia Empirica, sólo acepta como

\footnotetext{
2 ARISTÓTeles (1973). "Poética", 1451a/1451b. En: Obras Completas. Madrid: Aguilar, p. 85.

${ }^{3}$ Los métodos de recuperación y construcción, así como tipos y modelos han sido trabajados anteriormente en la concepción de la Historia de la Filosofía por: $\mathrm{M}^{\mathrm{a}}$ Isabel LAFUENTE (1986). Teoría Metodología de la historia de la Filosofía. Universidad de León: Contextos, $\mathrm{N}^{\circ} 4$.
} 
su forma propia la narración, y su característica principal es que el historiador no acepta subjetivamente seguir ninguna razón (ni lógica formal ni material, ni $a$ priori, ni a posterior) como rectora de ella; pero objetivamente la narración siempre resulta ser expresión de una determinada forma de razón filosófica, lo que supone un determinado orden lógico y su adscripción a una determinada forma filosófica (idealista, marxista, historicista, etc.). Este proceder puede adquirir la atractiva forma de una narración fabulosa, mítica, pero como sucede con los mitos, según ponen de relieve los trabajos de Levi-Strauss y Mircea Eliade, ${ }^{4}$ estas narraciones tienen un orden lógico; simplemente éste no se explicita. Su resultado es que de esta forma la Historia sólo genera y se apoya en la transmisión y en la memoria (no en la reflexión), siendo concebida como la memoria y la experiencia de la Humanidad, y como transmisión del pasado. Su principio es la imitación, no la producción; entiende el progreso de forma homogénea (temporalidad lineal), y su método es el de recuperación.

El método de recuperación, se basa en la necesidad de una previa comprensión de los hechos que de paso a su descripción. Esta metodología da lugar a una tradición histórico-erudita, que puede ser entendida perfectamente como filológica, y se centra en el examen de documentos cuyo sentido se trata de determinar según fue entendido por el autor. Se intenta mostrar lo que un texto o documento significa por él mismo, y de entender una época en función de los significados recogidos; supone un esfuerzo por encontrar y mostrar fuentes originales e incontaminadas y, en todo caso, de alcanzar a mostrar sus relaciones originales. La finalidad, u objetivo, que se propone es entender el pasado como pasado, desvelar su verdad oculta, pero esto resulta imposible si no es suponiendo un esquema conceptual existente en el pasado, de forma que el supuesto subyacente en este método resulta ser, según ya afirmaba Cohen:

"...que el esquema conceptual pasado existe y puede descubrirse en su integridad o en parte". 5

Cuando esta forma metodológica empirista se une a la historicista, se muestra que su principal característica es la de atender a los aportes de las prácticas

${ }^{4}$ Aparte de las Mitológicas I, II (1968 y 1982). México: F.C.E. y las Mitológicas III y IV (1970 y 1976). México: Siglo XXI, puede verse: Claude LÉVY-STRAuSs (1987). «La estructura de los mitos». En: Antropología Estructural. Barcelona: Editorial Paidós. Mircea Elíade (1968). Mito y Realidad. Guadarrama: Madrid.

${ }^{5}$ H. COHEN (1976). «Keeping the History of Philosophy». En: Journal of Philosophy, no 4, pp. 383-390. La misma tesis la mantienen los historiadores de los Annales, los positivistas y los estructuralistas. 
históricas desde una perspectiva subjetiva. No se entiende que el historiador tenga que buscar objetividad, sino al contrario poner de relieve la movilidad, la vitalidad y la temporalidad de lo humano, por lo que buscar lo objetivo es para esta orientación metodológica cosificar al sujeto. Unido a estos principios, el método de recuperación se caracteriza por negar posturas doctrinales, pues lo contrario supone admitir esquemas filosóficos previos, por lo que un historiador incardinado en esta forma de entender la Historia no aceptaría, por ejemplo, trabajar sobre el feudalismo, pues desde su perspectiva sólo existen los feudalismo, lo contrario es idealismo o marxismo y, en todo caso, Filosofía y no Historia. Este tipo de hacer y entender la Historia tiene como único recurso las fuentes, que se trata de revisar e interpretar, pero nunca de contrastar y utilizar como medio para la búsqueda de leyes.

\section{LA HISTORIA TOTAL Y GLOBAL: EL MÉTODO DE RECONSTRUC- CIÓN}

Las concepciones de la Historia opuestas a la concepción empírico-historicista arrancan del positivismo comtiano que introdujo una perspectiva de la Historia como ciencia que, frente a la anterior, aún sigue manteniéndose hoy, y sitúa el hacer histórico en sentido fuerte como el paso del mito, caracterizado por establecer relaciones cualesquiera entre cualesquiera hechos, a la búsqueda y determinación de leyes objetivas y con valor social. El positivismo solidario de una metodología cuantitativa, que se orientó inmediatamente a la formación de profesionales cuenta con autores tan significativos como B.G. Niebuhr, L. Ranke, H. Taine, R. Makckenzie, Ch. V. Langous, M. Seignobos. Otro tanto puede decirse de las concepciones marxistas y estructuralistas. Esta última, que tuvo gran auge entre los años 1960-1970, tiene entre sus representantes más conocidos historiadores como F. Furet, D. Richet, Robert R. Palmer, Jacques Godechot y Alfred Gobban.

Ahora bien, las tendencias más importante del siglo XX, que siguen influyendo en la producción y controversias de los historiadores, han sido, por una parte, la Escuela de los Annales y por otra la nacida de las posturas hermenéuticas de la Escuela de Frankfurt. Ambas tienen una característica común: afirman la necesidad del conocimiento histórico, pero sitúan el centro de lo histórico en la acción humana (sea del tipo de que sea). No es ya la vitalidad o la movilidad de lo humano lo que importa, sino la condición del hombre como protagonista de la historia.

La Escuela de los Annales, fundada en el año 1929 por Marc Bloch y Lucien 
Febvre $^{6}$, propugna una ampliación de los temas de estudio de la Historia de forma que estos dejan de ser exclusivamente políticos, bélicos y diplomáticos, para ser también científicos, culturales, religiosos, siendo su base de estudio el acontecimiento o evento. Durante la segunda y tercera etapa de la Escuela ha ido ampliándose el objetivo de la Historia, de forma que ha venido a entenderse que tiene que ocuparse también de temas antropológicos, de las ideas, de las mentalidades y de las representaciones, así como abrirse a esquemas críticos. Esta es la idea de Historia Total, denominación debida a P. Vilar, que tuvo y tiene gran éxito en ciertos grupos de historiadores en toda Europa e incluso de Norteamérica. ${ }^{7}$ La polémica en torno a si la realización de la historia tenía que ser social o económica, vino a dirimirse (al menos en ciertos sectores) al aceptar como criterio el enunciado por L. Febvre:

"No hay historia económica y social. Hay la historia sin más, en su unidad. La historia que es por definición absolutamente social" ${ }^{\text {. }}$.

Pero, realmente hoy ya no puede hablarse sin más de positivismo, en cuanto hay autores que consideran también positivista la orientación hacia el individualismo (Popper, por ejemplo); ni tampoco puede hablarse sin análisis de historiografía marxista, como cuando simplemente se entendía que esta concepción consideraba que la práctica era la base del hacer histórico (P. Villar) y, por lo mismo, tampoco de historiografía estructuralista. Hoy los planteamientos son mucho más complejos y en ellos se entrecruzan diversas líneas, por lo que a lo que hay que atender no es

${ }^{6}$ Ambos enseñaban en la Universidad de Estrasburgo, y estaban ligados a la publicación: Annales d'histoire économique et sociale, luego denominada: Annales. Economies, Sociétés, Civilisations, y a partir de 1994: Annales. Histoire, Sciences Sociales. La segunda generación (1945-1968) tiene como representante fundamental a Fernand Braudel, y la tercera (1968-1890) a Michel Foucault, Emmanuel Le Roy Ladurie y Jacques Le Goff, relevantes historiadores pertenecientes a esta tendencia que mantiene relación con importantes grupos de historiadores ingleses como los pertenecientes al grupo Past and Present: E. Hobsbawm, E.P. Thomson, R. Samuel, Ch. Hill, A.L. Morton, y posteriormente P. Burke. En Norteamérica la recepción de esta escuela dio lugar al ensayo de nuevos métodos como el cuantitativismo de la clitometría o new economic history (nueva historia económica), que tiene como principales representantes a los economistas de Robert Fogel y Douglass North. Entre los historiadores españoles esta tendencia historiográfica cuenta con autores tan importantes como Manuel Tuñón de Lara y Joseph Fontana.

${ }^{7}$ Cfr.: P. VILAR (1960). Croissance économique et analyse historique. Première conférence internationale d'histoire économique. Stockholm. París/La Haya: Mouton, 1960, pp. 41-85. En español: P. VILAR (1964). Crecimiento y desarrollo. Economía e historia. Reflexiones sobre el caso español. Barcelona: Ariel, 17-105.

${ }^{8}$ L. FeBVRe (1970). Combates por la historia. Barcelona: Ariel, p.39. 
sólo a su propuesta, sino a su resultado, no sólo a lo que se dice, sino a qué se hace, y a qué resulta de ello.

El principio básico de esta forma de entender la Historia es la producción, no entendida sólo en forma económica sino como operación fundamental de la acción humana. El ser humano, su actividad, su evolución, su intervención en los procesos de cambio (social, histórico y cultural) pasan a primer plano, y con ello el trabajo fundamental del historiador se centra en los acontecimientos y los problemas de su temporalización (largo, corto, etc.), y en la reconstrucción de los hechos (o construcción de los hechos). La Historia ya no se considera la experiencia o memoria de la humanidad, sino el lugar de los problemas que han afectado al ser humano.

El método que corresponde a esta forma de entender la Historia es el de reconstrucción, cuya primera característica es no aceptar como base la existencia de un supuesto esquema original del pasado, sino la necesidad de constituir por el historiador un esquema actual a partir del que realizar la búsqueda. Evidentemente este esquema resulta a priori, impuesto sobre el material pasado, pero no puede ser arbitrario, sino ligado a una doctrina que haga posible un proceso hermenéutico (comprensivo) y fenomenológico que no se quede en la mera interpretación, o descripción, sino que sea capaz de alcanzar la representación de los hechos.

Cuando a una narración se le concede capacidad de representación ésta va unida, como ya entendía Gueroult que pasaba con las doctrinas filosóficas, ${ }^{9}$ a su pretensión de verdad; pero, si se considera que ésta reside en el sentido de la verdad de los enunciados, es decir, en el orden de la génesis, y se entiende que lo histórico se distingue de lo sistemático filosófico y que éste pertenece al orden de la estructura, entonces la verdad sólo es formal, y la representación vacua, lo que permite aproximar la historia y la literatura. Ahora bien, es necesario señalar que es precisamente este principio, el de representación, el que tiene que permitir de forma efectiva distinguir entre una obra literaria y una histórica, pues si bien ambas, en cuanto formas de narración, pueden coincidir en su perfección dispositiva (lo que hace posible considerar a ambas como obras de arte), nadie exige a la obra literaria que represente una realidad, lo que sí corresponde necesariamente a la génesis de la reconstrucción histórica. Pero esto supone no negar, sino aceptar la necesidad de que la Historia tenga una estructura filosófica capaz de explicitarse en el orden genético, lo que requiere un proceder dialéctico

${ }^{9}$ Cfr.: M. Gueroult (1959). «The History of Philosophy as a Philosophical Problem». En: The Monist, 53, pp.585-586. 
que permita establecer una relación entre los principios aceptados (filosofía) y los principios negados (sombras, usando la terminología de E. Trías ${ }^{10}$ ), que haga posible lograr una aplicación de los procedimientos al material histórico que de paso una verdadera explicación histórica.

Evidentemente se puede decir que el esquema del pasado, como pasado, puede no participar de una doctrina previa, pero sí mostrar la verdad interna (inmanente) de las acciones, acontecimientos y hechos históricos. Esta forma de entender el problema, que tampoco deja de inscribirse en la vía estructuralista, sólo permitiría mostrar la verdad de la génesis de forma a-crítica respecto de sus fuentes, influencias. Si se procede así, tanto el orden estructural como el genético resultan imparciales respecto de la verdad representativa, pero se limitan a exponer un orden histórico homogéneo y/o heterogéneo que no pasa de constituir exposiciones por simpatía.

En las interpretaciones reconstructivas que siguen una forma doctrinal (idea), aquellas que no son meramente reproductivas (pues resultarían meras tautologías), se dirime de forma efectiva la verdad histórica y el alcance del sistema de partida, que puede resultar enriquecido o inoperante. Considero que ésta es la tarea, exploración y trabajo metodológico, propia del historiador que entiende que su labor tiene que permitir realizar aportaciones fructíferas al logro de nuevos principios que contribuyan a la mejor práctica y desarrollo de la Historia.

En la últimos años, las investigaciones en Historia están tomando un nuevo rumbo (no separado de la relación Historia-Social e Historia-Cultural, pero llegando a plantearse en la forma de Historia Global ${ }^{11}$ ) impulsado por las investigaciones sobre lo imaginario, noción que se está aplicando en tanto se entiende que los discursos históricos están siendo falseados y/o instrumentalizados en beneficio de la ideología dominante (poder) en una sociedad, de forma que el discurso histórico se ha vaciado de todo valor humano, por tanto, de todo interés general. ¿Qué se entiende por imaginario?

Esta noción, fue desde sus comienzos una noción muy controvertida, y se puede recordar al respecto la diferencia entre Levi-Strauss y el autor griego, porque

${ }^{10}$ E. TRíAs (1983). La Filosofía y su sombra. Barcelona: Seix Barral.

${ }^{11}$ Hay numerosa bibliografía sobre el tema. Un artículo interesante es el de José María IMízCOZ (2001). Introducción: Actores sociales y redes de relaciones: reflexiones para una historia global. Bilbao: Universidad del País Vasco, pp. 19-30. Su artículo (2004). «Actores, redes, procesos: reflexiones para una historia más global». En: Revista de la Facultad de Letras. Historia. Porto III, Série, vol. 5, pp. 115-140, esta disponible es http://ler.letras.up.pt/uploads/ficheiros/2378.pdf [Consultado 5-09-2010]. 
mientras aquél entendía que las significaciones imaginarias pertenecían a la estructura social, éste no concibió el imaginario de forma impersonal. Es cierto que Castoriadis concibe el imaginario como el magma de creación permanente de lo social, y entiende que toda entidad, significado o sentido, se crea (no se produce) de forma imaginaria. ${ }^{12}$ Igualmente sostiene también, que toda variación de una concepción vigente pasa por la formación de una imagen alternativa a la aceptada en un momento dado, y que la creación del nuevo significado nacido de dicha imagen tiene lugar en la sociedad como ente colectivo, impersonal y anónimo, pero la imagen, el significado y lo simbólico constituyen a lo colectivo como un sujeto ligado al deseo. ${ }^{13}$

Puede entenderse que los imaginarios forman parte del orden cultural, de las opiniones, mitos, leyendas, creencias, etc., y suele entenderse esta noción como próxima a la interpretación que Parsons hizo de las representaciones colectivas identificándolas con la realidad social, ${ }^{14}$ que dio lugar al problema de saber sobre las condiciones de identificación entre la realidad social y sus manifestaciones o representaciones. En efecto, el problema se plantea en cuanto, aún admitiendo que el imaginario es un magma, no puede negarse que el proceso de su formación supone una reacción del imaginario sobre los individuos a los que impone la forma de realizar la acción, de forma que la tesis del individualismo (humanismo clásico), que hacía descansar toda posibilidad de innovación en el individuo resulta inadecuada, pues las percepciones, sentimientos e iniciativas individuales son siempre encauzadas y reguladas desde el sistema de reglas no escritas en que consiste el mismo imaginario.

12 Castoriadis define la noción de magma frente a la teoría general de conjuntos clásica y la noción sistema tradicional; dice así: "Un magma es aquello de lo cual se puede extraer (o, en el cual se puede construir) organizaciones conjuntistas en cantidad indefinida, pero que jamás puede ser reconstruido (idealmente) por composición conjuntista (finita ni infinita) de esas organizaciones". C. CASTORIADIS (1975). La institución imaginaria de la sociedad, vol. 2. Barcelona: Tusquets, p. 288.

${ }^{13}$ No se puede entender que lo imaginario sea producido desde o a partir de un sujeto que intencionalmente contuviera todas las posibilidades; la sociedad no se instituye a partir de un núcleo dado con anterioridad que tienda a reproducirse, pero lo imaginario al constituir el modo de ser de los objetos, su significado, la red de significaciones derivadas, los dispositivos materiales que dan existencia a esos objetos, el comportamiento aceptable de los sujetos respecto de ellos, etc., proyecta una imagen de la sociedad, y le presta el carácter de un sujeto caracterizado por el deseo de satisfacer aquellas significaciones ausentes (faltan) en ella. De esta forma la sociedad no puede concebirse como un sujeto que reproduce su identidad o tiende a reproducirla, sino un sujeto que se crea, pues el logro del deseo no mantiene la identidad anterior incólume, sino que la transforma. Véase: C. CASTORIADIS, Ibid.

${ }^{14}$ Cfr.: T. PARSONS (1937). Las estructuras de la acción social. Madrid: Guadarrama, sobre todo p. 452. 
Ciertamente hoy ya no puede evadirse la consideración de lo imaginario social en la producción de lo real, como no puede negarse su lugar en la producción del orden social y su regulación hasta alcanzar su plasmación en la ley, pero la forma en que se atiende a su producción muestra la necesidad de sostener que el imaginario no sólo puede comportar opinión y/o creencia, sino que es necesario también una reflexión ${ }^{15}$, pues es evidente que, supuesto el deseo y la necesidad de cumplimentarlo, resulta ineludible responder a preguntas tales como: ¿por qué se produce este nuevo significado, o símbolo, o sistema de símbolos, y no otro?

La distinción entre imaginario instituyente (o constituyente) e instituido (constituido), lejos de resolver estos problemas los hace más patentes, por una parte porque nos remite a la consideración de los problemas ligados a la noción de poder ya examinados por M. Fouacult. Por otra parte, porque nos remite al tema del orden correcto, o sociedad correctamente ordenada de J. Rawls, que puede entenderse ligado exclusivamente al favorecimiento de determinados intereses, $\mathrm{y}$, por otra, porque, aunque Castoriadis sostenía que la psique individual no puede ser absorbida por lo imaginario social, pues psique y sociedad son inseparables pero irreductibles, de forma que la institución social del individuo solo va a abrir para éste:

\section{“...la posibilidad de hallar y de dar existencia para él a un sentido, en la significación social instituida", 16}

El análisis de lo imaginario desde el punto de vista de la consideración de la causalidad psíquica según fue realizada Michotte, muestra que esa absorción puede ser una realidad efectiva. ${ }^{17}$

Considero que estos problemas tienen que llevar a trabajar en el campo de investigación sobre los imaginarios considerándolos desde el punto de vista

${ }^{15}$ Castoriadis intentó mostrar de qué forma había que entender lo imaginario como condición de todo pensamiento, pero la forma en que desarrolla y se está desarrollando su concepción, no alcanza la formación conceptual, sino que permanece en la opinión o creencia. "Lo imaginario, por supuesto, también es condición de todo pensamiento, desde el más chato, el apenas pensamiento que se reduce a la manipulación mecánica de signos, si eso fuera posible, al más rico y el más profundo de los pensamientos". C. CASTORIADIS (1975). La institución imaginaria de la sociedad, vol. 2. Barcelona: Tusquest, p. 277.

${ }^{16}$ Ibid., p. 252.

${ }^{17}$ Este problema ha sido expuesto con mas detalle en M I. LAFUENTE (2007). «La educación universitaria hoy, desde la convergencia entre calidad e imaginarios sociales». En: Revista de Ciencias Humanas. Colombia: Universidad Tecnológica de Pereira, Año 12, Enero-Junio, pp.127146. 
constitutivo, es decir, ni como constituyente ni como constituido, sino en cuanto la imaginación da lugar a un orden dialéctico que ya no se puede apoyar en la simple creencia, sino en la reflexión sobre las prácticas y los significados y valores ligados a las acciones de los individuos y grupos sociales. De esta forma lo imaginario resulta sumamente importante para la investigación social, pues permite explicar los movimientos sociales según la forma en que se generan los vínculos o relaciones concretas, haciendo visible la invisibilidad social, de forma que la realidad histórica puede abordarse no sólo desde una relación de alteridad (como ya pusieron de relieve Hegel y Marx), sino también desde la visualización de aquello que se oculta, que resulta invisible en una sociedad. ${ }^{18}$ Pero, en este sentido lo imaginario, en todo trabajo, tiene que considerarse no como la razón (en este caso social), sino como un concepto empírico que exige siempre resolver el problema de la realidad del parecer, lo que supone el examen del parecer mismo (del imaginario), es decir, de lo que en una sociedad aparece como nación, o como mercancía, o como Dios.

En este contexto considero que no sólo los planteamientos causales ligados a las concepciones generalmente positivistas o estructuralistas son los que pueden proporcionar el marco epistemológico adecuado para los imaginarios, sino también los que se abren a planteamientos funcionalistas como puede ser el de M. Bunge, el de N. Luhman, o el de Zemelman, en tanto que permiten atender prioritariamente a las nociones de problema y de posibilidad.

Estas concepciones permiten considerar que la idea de Historia Total, deja de ser una idea finalista abstracta (un desideratum en todo caso, como entendía P. Vilar), y adquiere una forma determinada, pero compleja, pues es, por una parte, estructural (holista) y, por otra, funcional (organicista). Entender la Historia Total en concreto, supone entenderla en función de la construcción de un hecho y aceptar que esto exige conjugar ambos sistemas: todo/partes, sistema global/sistemas parciales, de forma que tenga lugar su reconstrucción total, por tanto, dar lugar a su explicación causal y mostrar funcionalmente que acontecimientos son visibles y cuáles resultan invisibles. Es la convergencia entre la explicación causal y la funcional la que puede permitir dar cuenta tanto del por qué este hecho, como de su significado y sentido.

${ }^{18}$ Considero que esta forma de entender el problema está muy cercana a la que en la Universidad de Santiago de Compostela está elaborando José Luis Pintos y los investigadores ligados al proyecto de investigación que se está desarrollando sobre diversos aspectos de éste tema, y sobre el que ya se han realizado varias publicaciones, tanto en editoriales como en Internet. 
De esta forma se solventaría, además, una de las dificultades principales planteadas por Habermas a Luhmann: que la distinción sistema/entorno llega a constituir todo sistema como parcial (autopoiesis y autoafirmación del sistema), independiente de todo otro, por lo que la apertura a lo posible concluye en una negación de la posibilidad filosófica más importante: la de establecer relaciones transcendentales. ${ }^{19}$

\section{LOS PROBLEMAS DE ORDEN Y CONEXIÓN EN HISTORIA}

Ya desde la Escuela de los Annales, pero sobre todo con la introducción de los imaginarios sociales, se produce la fusión entre Historia Social e Historia Cultural en la Historia Total y luego en la Historia Global, entendiendo que así puede conocerse la realidad que lleva a constituir o a deshacer realidades sociales como son las Instituciones, los hechos económicos y políticos, o las realidades culturales en su identidad o diferencia. Los principales problemas que ha suscitado esta fusión son los siguientes:

1. La tendencia a la reducción de todo conocimiento a creencia u opinión.

2. La producción de una literaturización, que si parece aceptable en cuanto ambas pueden coincidir en ser formas de ejercer una crítica, que permite poner de relieve las insuficiencias que afectan a un sistema, y motivar el deseo, sin embargo no puede establecer un concepto que permita variar la forma de concebir lo real y haga posible explicar la forma de variar su producción.

3. La tendencia a la unificación metodológica, de forma que el historiador prescinde de una metodología propia y se suma a las del sociólogo. En este sentido destaca el hecho de que la aceptación de los métodos cuantitivos/cualitativos lleva a diluir el conocimiento en la forma de creencias de la humanidad, y a entender que son éstas las que dan lugar y explican la aparición, desaparición o alternancia de instituciones, formas de poder, etc.

4. Se tiende a buscar y establecer conexiones (estas suelen ser tratadas como temas o problemas) entre diversos ámbitos, poniendo de relieve las diferencias e identidades existentes entre ellos, y buscando saber si dan lugar o no a una única estructura y por qué. Esta forma de proceder es común a la

19 J. HABERMAS (1989). «Excursus sobre Niklas Luhmann: apropiación de la herencia filosófica del sujeto en términos de la teoría de sistemas». En: HABERMAS, J. El discurso filosófico de la modernidad. Madrid: Taurus, pp. 434-453. 
Sociología y a la Historia, de forma que un historiador puede considerar la unidad de España a través de las conexiones que tienen lugar entre las vidas de Isabel y Fernando con la formación de la conciencia de identidad como unidad diferenciada, pero gestada en las formas políticas parciales que constituían sus antecedentes. E igualmente, un sociólogo puede considerar la importancia social de los viajes, a partir de las conexiones entre las vidas de dos mujeres, María ama de casa y Marta política de profesión, y la importancia que ese acontecimiento cobra en sus vidas.

La conexión de historias que fundan los imaginarios, cuando se lleva a cabo desde los imaginarios constituyentes o constituidos, da lugar a una nueva aproximación de la historia a la literatura y a la sociología, por tanto, a la entronización de mitos y al progreso homogéneo. Metodológicamente, o se mantiene la práctica de la historia técnico-filológica apoyándose en los documentos como determinantes sociales, políticos, religiosos, etc., o se desarrollan formas noveladas.

Cuando los imaginarios se entienden como constitutivos, la conexión adquiere otra forma, la que va ligada al método de reconstrucción. El trabajo entonces tiene lugar estructuralmente en dos niveles: en el primero se examinan las conexiones (relaciones o vínculos) que hacen posible establecer un todo ordenado. Los vínculos o relaciones constituyen partes de esa estructura, de esa totalidad ordenada, por ejemplo aquellos que socio-culturalmente permiten saber sobre la realidad de una Nación-Estado, como tales pueden considerarse por ejemplo, la educación, la economía, la religión, la política, etc. Pero este orden, para serlo realmente, tiene que incluir ya los principios y documentación que permitan dar cuenta de las historias alternativas, aquellas que han quedado ocluidas en su constitución $\mathrm{y}$, que para no adquirir la forma novelada, tienen que hallarse informadas mediante materiales, restos $\mathrm{y}$ documentos que puedan aportar significados distintos o variaciones en los significados.

En el segundo nivel se considera el todo como sistema en formación, que tiene que trabajar con aquellos vínculos que hacen posible las relaciones entre los hombres: códigos éticos (honor, sinceridad, responsabilidad, etc.), formas comunicativas, normas, reglas y promesas, sociales y políticas, hasta alcanzar los vínculos culturales efectivos (lengua, creencias, tradiciones, etc.).

El interés y oportunidad de esta forma de entender el trabajo en Historia lo pone 
de relieve Habermas, ${ }^{20}$ y podemos resumirlo en los siguientes puntos:

1. La Historia no puede entenderse sólo como la experiencia de la humanidad, sino también como el lugar de las expectativas.

2. Hay que concebir también la Historia, como la entendía W. Benjamín, cuando invertía la forma tradicional de entender la relación experiencia $=$ pasado, expectativa $=$ futuro, $y$ tener presente que en todas la épocas pasadas existe un horizonte de expectativas no satisfechas, que la actualidad orientada al futuro va a encontrar en la forma de revivencia que tiene que satisfacer.

3. La actualidad tiene una deuda contraída con el pasado: la de desarrollar las expectativas que fueron contraídas en el pasado.

4. La tradición comprende tanto la barbarie como la cultura.

\section{LAS ORIENTACIONES METODOLÓGICAS TIPOLÓGICA Y FUNCIONAL: TIPOS Y MODELOS.}

\subsection{Tipos}

Hoy cuando el historiador se plantea los modos de hacer y no sólo de concebir la Historia, se encuentra con la necesidad de trabajar tanto con métodos cuantitativos como cualitativos, lo que se muestra, claramente y desde el primer momento, en cuanto se intenta construir un hecho y su correspondiente conocimiento histórico, es decir, en la simple aproximarnos a un material que tiene que ser ordenado. Supongamos que estamos trabajando con la noción de Nación, y el historiador se encuentra con documentos, textos etc., que le muestran que ésta se considera desde una multiplicidad diversa de perspectivas, como por ejemplo: voluntad general, identidad colectiva, comunidad ficticia (política). Evidentemente en este caso la primera tarea será buscar aquella documentación que le proporcione una información mediante la que sea capaz de producir un orden. Éste puede obedecer a la búsqueda de aquellos fundamentos que soporten dichas perspectivas: sustantivo, subjetivo, imaginario, cultural. De esta forma, la primera forma de orden es realizar una clasificación de las distintas perspectivas en que la noción de nación resulta concebida según la pluralidad de principios que se muestren como base de las perspectivas, dando lugar a establecer aquellas clases a las que las

${ }^{20}$ J. HABERMAS (1989). «Excursus sobre las tesis de Filosofía de la Historia de Benjamín». En: HABERMAS, J.: El discurso filosófico de la modernidad, Madrid: Taurus, pp. 23 y ss. 
perspectivas pertenezcan como tipos individuales. La siguiente clasificación puede ser una muestra:

\begin{tabular}{ll}
\multicolumn{1}{c}{ Clases } & \multicolumn{1}{c}{ Tipo Individual } \\
\hline 1. Sustantivo & Voluntad general \\
2. Subjetivo & Identidad colectiva frente a otra/s \\
3. Imaginario & Comunidad ficticia (política) \\
4. Cultural & Tradiciones (tradición alemana, tradición francesa, etc.) \\
\hline
\end{tabular}

La condición de pertenencia a las clases de las perspectivas como tipos individuales, se muestra muy clara, pero a una clase pueden pertenecer como tipos individuales otros de otra u otras clase; por ejemplo a la clase de las naciones determinada por el principio sustantivo, pertenece también como tipo individual aquellas que siguen la tradición francesa, lo que muestra la posibilidad de combinaciones de tipos.

Los tipos individuales, pueden ser agrupados según lo que Von Mises denomina tipos reales, ${ }^{21}$ es decir, tipos genéricos, lo que supone agrupar tipos individuales según rasgos inmediatos de ellos, y exige una recombinación de tipos individuales, por ejemplo, en función de un tipo complejo imaginario-cultural, que permita combinar una información inicial con la pueda surgir, por ejemplo, de la obtenida relativamente a la condición de la nación como entidad política. Si consideramos la idea de nación según la clase imaginario, y su tipo individual comunidad política, podemos establecer los siguientes cuatro tipos genéricos, tipos individuales y casos concretos.

Tipos Genéricos:

1. Naciones-Estado: Estados uni-nacionales (Ej. Japón) y estados plurinacionales cuyos miembros pertenecen a entidades colectivas diferentes (Ej. España, Bélgica).

2. Naciones sin Estado (Ej. Cataluña, Québec).

3. Estados sin Nación (Ej. Mónaco).

4. Naciones divididas en más de un Estado (Ej. Corea)

21 “..."real types", that is, class concepts distinguishing people or institutions according to nearly traits... This kind of classification is independent of specific understanding. It points towards something that in common to all members of the class". L. VON MISES (1958). Theory and History. Londres: Jonathan Cape, p. 315. 
Ciertamente la noción de tipo encierra numerosos problemas a la hora de trabajar con ella; vamos a considerar una forma de proceder en la que nos atenemos al planteamiento de Lazarsfeld que muestra el concepto de tipo ligado al de sistema descriptivo, lo que tienen la virtud de permitir tratar con un conjunto de datos cualitativos que van:

“...desde la simple enumeración de un conjunto de tipos desprovistos de relaciones lógicas hasta las tipologías sistémicas, en las cuales cada tipo representa una combinación particular de atributos fundamentales". ${ }^{22}$

Lazarsfeld, tomando como punto de partida el trabajo en que Hempel y Oppenheim analizan el concepto de tipo desde un punto de vista lógico, introduce el concepto de espacio de atributos y tres operaciones a las que denomina reducción, sustrucción y transformación. ${ }^{23}$ El punto de partida de cualquier análisis del concepto de tipo reside en el reconocimiento de que ese concepto se refiere siempre a ciertos compuestos de atributos. Podemos suponer, para nuestro caso, tres atributos $\mathrm{X}, \mathrm{Y}, \mathrm{Z}$, asociados a tres ejes $\mathrm{x}, \mathrm{y}, \mathrm{z}$, respectivamente. Si un objeto posee esos atributos tendrá que tener su marca positiva (es decir en el eje positivo), si no es así, tendrá que ser marcado en el eje negativo (su marca será negativa). Estas marcas en los ejes constituyen las coordenadas que permiten establecer el espacio definitorio del objeto. De esta forma lo que se realiza es una operación clasificatoria cuyo resultado es reagrupar varias combinaciones en una clase o tipología. ${ }^{24}$ Suponiendo el caso de los tres atributos mencionados, tendremos ocho combinaciones posibles, que nos permitirán establecer el espacio de atributos en el que se podrán determinar los diferentes tipos según las combinaciones de atributos concretas que tengan lugar en él. La representación abstracta de dicho espacio sería:

$\begin{array}{cccc} & \mathrm{X} & \mathrm{Y} & \mathrm{Z} \\ 1 & + & + & + \\ 2 & + & + & - \\ 3 & + & - & + \\ 4 & + & - & -\end{array}$

${ }^{22}$ P. F. LAZARSFELD (1970). «Sur les téchniques de construction de typologies dans les sciences sociales». En: Paul Felix LAZARSFeld Philosophie des sciences sociales, Paris: Gallimard, pp. 361374.

23 "Reduction, substrucion et transformation peuvent éter disignées comme des "operations typologiques" parce qu'elles permetten de lier tout système de types à un espace d'attributs". Ibid., p. 374.

${ }^{24}$ Ibid., p. 329 


$\begin{array}{llll}5 & - & + & + \\ 6 & - & + & - \\ 7 & - & - & + \\ 8 & - & - & -\end{array}$

La operación de reconstruir el espacio de atributos subyacente a un sistema topológico la llama Lazarsfeld substrucción, y como no puede decirse que para cada tipología exista un solo modo de reducción, y un solo espacio de atributos, pueden existir varias substrucciones. Se habla de de transformación en cuanto los diferentes espacios de atributos son mutuamente transferibles:

"Desde el punto de vista lógico, interpretar significa referir, por susbstrucción, a una tipología un espacio de atributos diferente del que una primera reducción lo había extraído, y buscar qué reducciones permiten reconstruir el sistema de tipos de ese nuevo espacio". 25

Transformación, reducción y sustrucción, son operaciones tipológicas que permiten asociar a todo sistema de tipos un espacio de atributos. A partir del planteamiento de este autor, una tipología puede entenderse como un sistema combinatorio de tipos posibles que sin embargo mantienen una unidad en cuanto todos coinciden en el mismo espacio de atributos, lo que permite proceder de forma distinta del mero empirismo, en cuanto cualquier tipología obtenida empíricamente puede ser referida a su espacio de atributos y, relativamente a sus posibilidades combinatorias, constituirse en parte de una totalidad ordenada. Es posible, también, suponer diferentes espacios de atributos que, mediante reducciones diferentes, produzcan las mismas tipologías.

Si se entiende que proceder según un orden lógico y combinatorio en la construcción de tipos solo conduce a establecer una tipología, como suelen mantener los defensores de la constitución empírica de tipos, se da lugar a una lucha vacua, puesto que ésta se pueda formular o que sólo se logre establecer un orden entre clases, depende muchas veces no sólo de la forma en que se proceda en su construcción, sino también del material existente.

Vamos a poner un ejemplo de construcción tipológica tomando dos formas de gobierno: Monarquía/ República, y construir su espacio de atributos relativamente a tres términos: Poder Político (institucional), Parcialidad Política, Constitución. El espacio sería el siguiente:

${ }^{25}$ Ibid., p.373. 


\begin{tabular}{ccccl}
\hline & $\mathrm{x}$ & $\mathrm{y}$ & $\mathrm{z}$ & \\
\hline & $\begin{array}{c}\text { Poder } \\
\text { político }\end{array}$ & $\begin{array}{c}\text { Parcialidad } \\
\text { política }\end{array}$ & Constitución & \\
\hline 1 & + & + & + & Rep. Presidencialista \\
2 & + & - & - & Rep. Totalitaria \\
3 & - & + & + & Rep. Parlamentaria \\
4 & - & + & - & Inconsistente \\
5 & + & - & - & Monarquías Híbridas \\
6 & + & - & + & Monar. Absoluta, Tiranía \\
7 & - & - & - & Monar. Parlamentaria \\
8 & - & & & \\
\hline
\end{tabular}

Evidentemente, este ejemplo, que construye dos formas tipológicas, puede complicarse mediante la puesta en relación de más atributos. Pero, en la forma que se presenta, los tipos resultantes tienen la característica de permanecer incomunicados, de forma que tan sólo pueden dar lugar, en todo caso, a un orden jerárquico.

La orientación metodológica funcional, que introduce la posibilidad de elaborar no ya taxonomías tipológicas, sino modelos, es ciertamente la que hace posible interpretar las formas sistemáticas dadas históricamente (las taxonomías por ejemplo), como funciones de varias variables. Esta última orientación, que es la que está hoy está teniendo más éxito, tiene como condiciones: establecer unos esquemas básicos (pueden ser tipologías, o dar lugar a ellas) que permitan la ordenación sistemática de las diversas ideas históricas (políticas, sociales, institucionales, éticas, etc.), y un sistema de operaciones que haga posible mostrar la posibilidad de transformación de unos sistemas en otros.

De esta forma, el modelo exige la interpretación de tipos y taxonomías, pero esto no significa que la vía funcional subsuma sin más la tipológica, pues la orientación que la historia adquiere en ambas formas de trabajo es distinta: mientras la primera tiende a presentar la historia como presidida por la idea de recurrencia, la segunda tiende a presentarla como unitaria.

\subsection{Modelos}

Metodológicamente los modelos son un intento de dar cuenta de las diferentes formas de temporalidad, implícitas en la comprensión histórica, que incide en el hacer del historiador de muy diversas maneras. La más tradicional, ligada al método de recuperación y a la tesis del conocimiento como representación (o reflejo) de los hechos, plantea este problema en cuanto se pregunta por la existencia de la inconmensurabilidad entre hechos y conocimiento (expresado en la 
narración), es decir, de cómo dar cuenta del espacio-tiempo existente entre el acontecimiento y el hecho histórico (reconstrucción-narración), que de no ser resuelto tiene como consecuencia la imposibilidad de hablar de realidad y de verdad en la Historia (la verdad sólo sería formal en todo caso), pues nada podrá dar cuenta de la relación entre la narración y el pasado. La historia cuantitativa aborda el problema entendiendo que la transformación de la metodología de la Historia en la actualidad, corre pareja con la revolución tecnológica y técnica que, solidaria de una nueva crítica documental, supone que ni datos ni documentos existen por sí mismos, sino en relación con la serie en que se inscriben, que tiene que contar con la que les precede y la que les sigue, como señala Furet:

"El documento y el dato ya no existen por sí mismos, sino con relación a la serie que los precede y los sigue; es por su valor relativo que se vuelven objetivos y no con relación a una impalpable sustancia "real". Y de este modo, el viejo problema de la "crítica del documento histórico se halla al mismo tiempo cambiado de posición. La "crítica" externa ya no se establece a partir de la credibilidad basada en la comparación con textos contemporáneos de otra naturaleza, sino a partir de la coherencia con un texto de la misma naturaleza, situado de manera distinta en la serie "temporal", es decir, antes o después. La "crítica" interna se encuentra tanto más simplificada cuanto muchas operaciones se "limpieza" de los datos pueden ser colocados en la memoria de una computadora". 26

La formalización del documento, en las nuevas tecnologías de la información (TICs), es la que instituye la coherencia; es un programa instalado en el ordenador el que permite saber sobre la existencia en un período largo o corto, y en cada unidad de tiempo sobre la frecuencia de los datos y de las mismas sucesiones lógicas. Pero, esto no elimina los problemas ni epistemológicos ni metodológicos; ni en lo que afecta a los sistemas de recolección automática de datos (el programa hay que hacerlo), ni respecto a la tarea del historiador. En lo que afecta a la selección automática de datos, porque no sólo es necesario afinar los sistemas de eliminación de errores, sino también porque se requiere solventar la existencia de límites en la cuantificación integral de datos, lagunas o series incompletas, etc.; en lo que afecta a la tarea del historiador, porque las nuevas tecnologías obligan al historiador a reflexionar sobre su forma de entender la Historia, de formular hipótesis, pues el historiador tiene que decidir sobre la pertinencia del uso de los

${ }^{26}$ F. FURET, F.(1971). "La historia cuantitativa y la construcción del hecho histórico" En: Historia Agenda. Selecciones. Pág. Web: http://www.cch.unam.mx/historiagenda/6/index.htm. [Consultada el 8/10/2008], p. 3. También: F. FuRET (1985). «Lo cuantitativo en historia». En: J. LE GofF y P. NoRA (Direc.): Hacer la historia. Volumen I: Nuevos problemas, Barcelona: Laia, pp. 55-73. 
documentos y fuentes para construir un hecho, sobre la organización de las series con relación a su cuantificación, sobre el sentido unívoco o no de éstas, etc. La codificación de los datos que permiten las TICs, hace más acuciantes una reflexión continua sobre la teoría y metodología de la Historia (lo que se muestra en la abundante bibliografía sobre el tema en los últimos 30 años), pues supone por parte del historiador la necesidad de tomar conciencia de que no existe una objetividad histórica oculta, que el tiene que descubrir, sino que lo visible y lo invisible, lo oculto y lo objetivo depende de la información que él posee, y de la forma en que construye los hechos en función de las hipótesis que sus conocimientos le permiten formular, como señala Furet:

“...el historiador ya no puede escapar a la conciencia de que ha construido sus "hechos", de que la objetividad de su investigación viene no solamente del empleo de procedimientos corrector en la elaboración y el procesamiento de tales "hechos", sino de la pertinencia con relación a las hipótesis de su investigación". ${ }^{27}$

La Historia no la hace el archivo de la memoria en que se ha transformado el ordenador. Un programa puede proporcionar un número muy alto de series repetitivas, la lógica del programa le puede permite conocer su coherencia e incluso su significado, pero esto no elimina la existencia de elementos históricos que no pueden registrarse en un programa: vestigios huellas, ruinas, y diversas formas icónicas, ni elimina la necesidad de establecer el criterio que hace necesaria la elección de una sucesión de datos con preferencia a otra (cuando además puede existir una equivalencia suficiente entre ellas) y establecer o trabajar un acontecimiento más bien que otro.

Aceptando que el historiador trabaja sobre problemas, como se tiende a pensar hoy a partir de la Escuela de los Annales, no se puede obviar que uno de los principales, porque afecta a su hacer, es el de saber (tanto si se procede en forma serial, y el historiador se limita a narrar series de datos sin ligarse a hipótesis alguna, sino simplemente a ponerlos de manifiesto ${ }^{28}$ como si el historiador se liga al procedimiento de establecer una hipótesis según la que elegir y/o ordenar las series) si es posible, y cómo, la continuidad en la historia, pues en uno o en otro caso (sea la seriación que siempre será particular, sea la elección de datos que siempre recaerá sobre un o unos acontecimientos y no otros), el caso es que la continuidad se muestra como un efecto que se constituye en la narración y que, por

${ }^{27}$ Ibid.

${ }^{28}$ Forma en la que procede el historiador que entiende que tiene que poner de manifiesto sólo lo que sucede, o lo que irrumpe como caso único (individual.) 
tanto, puede ser una ficción. Por ello, resulta preciso, por lo menos, saber en la forma que se puede proceder respecto de la relación discontinuidad/continuidad.

Serres al estudiar la historia de la matemática pone en relación la noción de continuidad con la de conexión y recurrencia, y estableciendo cuatro tipos de modelos:

"Hasta el momento hemos encontrado cuatro conceptos básicos: la historicidad propia de las ciencias (matemáticas) podía ser conexa y (o) discontinua; podía ser leída... en el sentido directo de la teleología o en el sentido inverso de la recurrencia. Habría en una primera aproximación cuatro tipos de modelos elementales: conexos directos y recurrentes, no conexos recurrentes y directos". 29

La oposición modelos conexos/modelos recurrentes, puede cruzarse con la de modelos unitarios/modelos recurrentes, manteniendo que la historicidad de aquella puede ser continua o discontinua, lo que hace necesario intentar entender los términos de estas oposición y la forma de su relación. Una estructura recurrente es una seriación de datos caracterizada por su repetición, puede decirse que lo que se repite es su forma o estructura típica (un tipo, por ejemplo una forma de gobierno), mientras que si se habla de estructura unitaria de la Historia se entiende que ésta es irrepetible, teleológica (tiende a un fin). Si la teleología se considera relativamente a procesos o períodos concretos, podrá mostrarse la repetición de ciertos patrones, que pueden coincidir con períodos en la Historia o ser invariantes en procesos históricos, pero siempre será privilegiado un patrón que como foco centrará la unidad histórica.

Epistemológicamente la oposición entre los términos continuidad/ discontinuidad al considerarse relativamente a la Historia se intentaba resolver tradicionalmente relacionándolos con nociones como ruptura, corte, génesis, etc., pudiéndose señalar autores claramente partidarios de la segunda como Bachelard, Foucault, Althuser, etc., o de la primera, como Comte, Duhem, Brunschvicg, pero también se aceptaban ambas posturas simultáneamente, como cuando se admitía la discontinuidad de las estructuras y la continuidad de los problemas.

Ahora bien, el problema de fondo lo plantea la confusión que la noción de continuidad introduce al intentar ser aplicada a la Historia, pues realmente sólo puede ser definida exactamente en matemáticas. ${ }^{30}$ En Historia no es factible

${ }^{29}$ M. SERRES (1968). Hermes ou la communication. París: Editions de Minuit, p. 95.

${ }^{30}$ La continuidad es una propiedad matemática que, cuando se entiende referida a funciones tiene la virtud de expresar la correlación de los valores particulares de una variable independiente y los de 
establecer una forma exacta de entender la continuidad, porque su fuente principal y su vehículo es el lenguaje que, caracterizado por su discreción simbólica, no es un lugar válido para su consideración matemática. Ahora bien, si examinamos el caso de la construcción del espacio de atributos y de la tipología resultante que hemos realizado, se muestra que existen ciertos tipos que son inconsistentes en ese espacio de atributos y, que al igual que sucede en las estructuras componentes de un sistema, constituyen sus restricciones, lo que da lugar a la consideración de su discontinuidad, por tanto a entender (y tener que atender a) sobre alternativas, invisibilidades o falta de documentación. Esto parece conducirnos a entender que la discontinuidad es la forma operatoria propia de la Historia, pero como podemos suponer que tipos y estructuras son transformables, obligadamente tenemos que preguntarnos cómo considerar estas relaciones si hemos supuesto que son discontinuas, pero resulta que podemos operar con ellas según transformaciones continuas.

Considero que planteados los tipos y estructuras en forma lógica, y siendo éstas las que permiten realizar trasformaciones que muestren como idénticas determinadas estructuras, se puede llegar a afirmar que la discontinuidad es la forma del producto, del resultado de lo hecho sobre los materiales (documentos, vestigios, etc.), y la que la continuidad pertenece, en todo caso, a la condición epistemológica, es decir, al segundo nivel en que el historiador se enfrenta al material como símbolo y en el que se hallan presentes tanto ideas, como problemas, como estructuras. Entonces parece de nuevo que es sólo el conocimiento ligado a la narración el que permite la continuidad, lo que requiere para su solución saber si entre ambos niveles existe conexión o no, ${ }^{31}$ es decir, que

la función. Así, por ejemplo, dada una función cualquiera $y=f(x)$ y un valor particular $x_{0}$ de la variable independiente $\mathrm{x}_{1}$, “... si la función representa un proceso continuo, entonces a valores de $\mathrm{x}$ que difieran sólo ligeramente de $\mathrm{x}_{0}$ corresponderán valores de la función $f(x)$ que difieren solo ligeramente del valor $f\left(x_{0}\right)$ en el punto $x_{o}$. Así, si el incremento $x-x_{o}$ de la variable independiente es pequeño, el incremento correspondiente $f(x)-f\left(x_{0}\right)$ de la función será también pequeño... si el incremento de la variable independiente... tiende a cero, entonces el incremento... de la función debe aproximarse a cero, hecho que puede expresarse del modo siguiente:

$$
\begin{gathered}
/ 1 / \lim \left(\mathrm{f}(\mathrm{x})-\mathrm{f}\left(\mathrm{x}_{0}\right)=0 .\right. \\
\left(\mathrm{x}-\mathrm{x}_{0}\right) \rightarrow 0
\end{gathered}
$$

La función $f\left(x_{0}\right)$ se dice continua en el punto $x$ si se verifica la igualdad $/ 1 / \ldots$ (y) continua en el intervalo dado si es continua en todo punto... de este intervalo; esto es, si se verifica la igualdad /1/ en cada uno de sus puntos". A. D. Alexandrov, A. N. Kolmogorov, M. A. Laurentiev (y otros) (1973) La matemática su contenido, métodos y significado". Madrid: Alianza Editorial, vol. 1, p. 118.

${ }^{31}$ Vemos que Serres entiende la continuidad como una propiedad que puede estar relacionada o no con la conexión, y es que la conexión es una propiedad de las relaciones que puede estar relacionada con ella, o ser contraria a ella en alguna forma. En efecto, la conexión puede ser contraria 
lo fundamental para la Historia Total es saber si entre estos dos niveles: el del producto (resultado) y el cognoscitivo existe alguna forma de conexión, y si la forma que adquiere ésta permite hablar de continuidad o no.

Conforme con los modelos ya distinguidos podemos establecer la siguiente tabla:

\begin{tabular}{lll}
\multicolumn{3}{c}{ Modelos } \\
\hline A. Conexos & B. No-Conexos \\
\hline I. Unitarios & Unitarios Conexos & Unitarios No-Conexos \\
II. Recurrentes & Recurrentes Conexos & Recurrentes No-Conexos \\
\hline
\end{tabular}

Como el producto que el historiador obtiene supone su trabajo sobre un material al que aplica una determinada metodología, la conexión que permite establecer la relación entre el orden ontológico-metodológico y el epistemológico (por tanto saber sobre la realidad en la historia), sólo se hace patente en el nivel en que la historia se hace filosófica, es decir, que saber sobre la conexión entre ambos niveles sólo es posible si la reconstrucción se realiza desde una idea filosófica; pero los modelos no-conexos pueden dar lugar a interpretaciones desde normas o reglas sociales, políticas, culturales en general, y dar lugar a saber sobre las formas que prevalecen en un momento en esos ámbitos.

De esta forma, es preciso afirmar que mientras en un modelo unitario-conexo la historicidad tiene que considerarse continua dado que las formas siempre tienden a confluir en un sistema final, aunque las formas típicas (seriadas) sólo se repitan en ciertos tramos, (tales son las formas de hacer en Historia que trabajan con las ideas de igualdad, libertad, perfección, y que permiten considerar la formación de una nación desde ellas (Ilustrados, Hegel, etc), los modelos unitarios no-conexos suponen la irrepetibilidad (no recurrencia) de las formas culturales, y son característicos de los trabajos en historia a partir de nociones de sociología, política, etc., que consideran la idea de nación desde esquemas sociológicos (ej. emigración, cambios de poder, etc.), así como de historia literaria y técnica. Estos dos son los casos extremos en lo que afecta a la continuidad y la discontinuidad, mientras que los otros dos modelos dan lugar a consideraciones intermedias.

a la universalidad de la relación, dado que una relación definida en una clase puede ser universal, es decir, aplicable a todos su miembros, pero no conexa (aplicable a cada "par", "terna", etc.), como sucede cuando se define la propiedad de "paralelas" de las rectas en el plano, donde resulta que ésta propiedad pertenece a las rectas del plano, pero cualesquiera rectas en él no tienen por qué ser paralelas entre sí. 
Los modelos recurrentes-conexos suponen recurrencia en la forma misma del desarrollo histórico, siendo característica principal suya permitir la recurrencia unida a la direccionalidad. Se sostiene que es la reflexión la que permite ordenar las formas anteriores a las posteriores, por lo que es el caso en que la historia se entiende como la búsqueda del origen y pervivencia de una idea (se trataría, por ejemplo, de estudiar la evolución de una idea de nación a lo largo de la historia, o de la idea de nación como capaz de dar cuenta de la direccionalidad de la historia) $\mathrm{Y}$, por último, los modelos recurrentes no-conexos tienen lugar cuando se trabaja con tipos (no con estructuras), que se consideran relacionados con sistemas culturales globales que aquéllos caracterizan por atender a los procesos históricos desde lo sensorial, lo ideal o lo ideativo, es decir, desde nociones que permiten poner de relieve la multiplicidad y la variedad que afectan a lo global.

Tipos y modelos permiten mostrar la altura que se ha logrado en el conocimiento de la realidad histórica: de la condición científica, política, económica, filosófica, artística, social, ética, de mentalidad, y de estilo de vida en general. Relativamente a la idea de nación permiten trabajar con ella como aquella forma política exigida, o negada, por una población o poblaciones en un contexto cultural restringido o general, y a saber sobre la pluralidad de condiciones y necesidades que han llevado en la Historia de la Humanidad a constituir esta idea, así como a la pluralidad de formas que ha adquirido y las ideas que han generado. Trabajo previo y necesario para saber qué valores defender.

\section{BIBLIOGRAFÍA}

ARISTÓTELES (1973). Obras Completas. Madrid: Aguilar.

ELÍADE, M. (1968). Mito y Realidad. Guadarrama: Madrid.

CAstoriadis, C. (1975). La institución imaginaria de la sociedad. Vols. 2. Barcelona: Tusquets.

CoHEN, H. (1976). «Keeping the History of Philosophy». En: Journal of Philosophy, ${ }^{\circ} 4$, pp. 383-390.

FebVRE, L. (1970). Combates por la historia. Barcelona: Ariel.

FURET, F. (1985) «"Lo cuantitativo en historia». En: J. LE GOFF y P. NORA (Direc.): Hacer la historia. Volumen I: Nuevos problemas Barcelona: Laia, pp. 55-73.

FURET, F. «La historia cuantitativa y la construcción del hecho histórico» En: Historia Agenda. Selecciones. Pág. Web: http://www.cch.unam.mx/historiagenda/6/index.htm. [Consultada el 8/07/2008].

HABERMAS, J. (1989). El discurso filosófico de la modernidad. Madrid: Taurus.

ImízCOZ, J. M (2001) Introducción: Actores sociales y redes de relaciones: reflexiones para una historia global. Bilbao: Universidad del "País Vasco, pp. 19-30.

LAFUente, I. (1986). Teoría y Metodología de la Historia de la Filosofía. Universidad de León: Colección Contextos, $\mathrm{N}^{\circ} 4$. 
LAFUENTE, I. (2007). «La educación universitaria hoy, desde la convergencia entre calidad e imaginarios sociales». En: Revista de Ciencias Humanas. Colombia: Universidad Tecnológica de Pereira, Año 12, Enero-Junio, pp.127-146.

LeVY-STRAUSS, C. (1987). Antropología Estructural. Barcelona: Editorial Paidós.

LAZARSFElD, P. (1970). Philosophie des sciences sociales. París: Gallimard.

MiSES, L. von (1958). Theory and History. Londres: Jonathan Cape.

PARSONS, T. (1937). Las estructuras de la acción social. Madrid: Guadarrama.

SERRES, M. (1968). Hermes ou la communication. París: Editions de Minuit.

VILAR, P. (1960). Croissance économique et analyse historique. Première conférence internationale d'histoire économique. Stockholm. París/La Haya: Mouton, 1960. En español: VILAR, P. (1964). Crecimiento y desarrollo. Economía e historia. Reflexiones sobre el caso español. Barcelona: Ariel. 